\title{
Application of Atomic Sparse Decomposition to Feature Extraction of the Fault Signal in Small Current Grounding System*
}

\author{
Nanhua Yu ${ }^{1}$, Rui Li $^{1}$, Jun Yang ${ }^{2}$, Bei Dong ${ }^{2}$ \\ ${ }^{1}$ Electric Power Research Institute of Guangdong Power Grid, Guangzhou, China \\ ${ }^{2}$ School of Electrical Engineering Wuhan University, Wuhan, China \\ Email: 793623451@qq.com
}

Received January, 2013

\begin{abstract}
Applying the atomic sparse decomposition in the distribution network with harmonics and small current grounding to decompose the transient zero sequence current that appears after the single phase to ground fault is occurred. Based on dictionary of Gabor atoms and matching pursuit algorithm, the method extracts the atomic components iteratively from the feature signals and translated them to damped sinusoidal components. Then we can obtain the parametrical and analytical representation of atomic components. The termination condition of decomposing iteration is determined by the threshold of the initial residual energy with the purpose of extract the features more effectively. Accordingly, the proposed method can extract the starting and ending moment of disturbances precisely as well as their magnitudes, frequencies and other features. The numerical examples demonstrate its effectiveness.
\end{abstract}

Keywords: Small Current Grounding System; Fault Line Selection; Atomic Sparse Decomposition; Matching Pursuit; Damped Sinusoids

\section{Introduction}

The small current grounding system include ungrounded neutral system, arc suppression coil compensated neutral system and high resistance-grounded neutral system. In our country, scientists and engineers have done a lot of researches on fault line selection in small current grounding system, a variety of line selection methods were put forward and some successes were achieved. But the problem of fault line detection hasn't been well settled because of that the fault current is small, the fault features are unobvious, the fault circumstances are complex and the mode of the system runs various. This will hinder the smooth development of power distribution automation system, damage the security and stability of the power system.

Fourier transforms and wavelet transforms are examples of time-frequency signal decomposition that have been used for many years. The Fourier basis provided a poor representation of functions well localized in time, and wavelet basis are not well adapted to represent functions whose Fourier transforms have a narrow high frequency support. In both cases, it is difficult to detect and identify the signal patterns from their expansion coeffi-

\footnotetext{
*This work is supported by Key Technology Projects of China South-
} ern Power Grid (No. K-GD2012-343) cients for the information is diluted across the whole basis. To extract information from complex signals, it is often necessary to adapt the time-frequency decomposition to the particular signal structures. [1]

Domestic and foreign scholars have proposed a variety of methods about fault line selection. Existing line selection method of stable state quantity [2-4] is difficult to meet the site operational requirements. There is still fault transient detection method [5-12]. The paper [5] proposes a distribution network fault line selection method about fusion of multiple sampling points' poll results based on S-transform through studying S-transform to extract the amplitude and frequency characteristic and phase-frequency characteristic of signal. This method needs to collect to the correct the feeder phase angle and frequency information. The paper [6,7] extract fault line travelling wave through using wavelet transform and structure criterion in order to determine fault line. Although wavelet transform has good enough time domain - frequency domain localization features to provide characteristics of the signal in different scales, it has ineffective application as it is vulnerable to the effects of noise. The paper [8] realizes the fusion line selection method through introducing the concept of fault measures and using Dempster-Shafer theory. The paper [11] captures feature band by comparing transient zero-sequence cur- 
rent amplitude and gets characteristic band signal through filtering. References[9,10] use wavelet packet to extract the transient signal, determined band which the transient capacitive current most concentrate by the principle of maximum energy and elected fault feeder. However, the feature band that can be used for line selection is influenced by the system parameters, failure modes and so on. Various feeders transient capacitive current concentration of energy bands are not the same, if the band is selected improperly, it is easy to make the fail selection of the fault line.

The paper [12] uses S-transform to process zero sequence current of each feeder, and determines the dominant frequency of the capacitive current by comparing transient energy of different frequency points, and selects the fault line according to the size of the energy. S-transform is the development of continuous wavelet transform and the short time Fourier transform, and has good time-frequency characteristic, but amount of information is so much after decomposition. As well as there is some line selection methods which combine steady state with transient state, but neural network algorithm exists local optimum problem, poor convergence, longer training time and limited reliability.

To compensate for these shortcomings, this paper provides an algorithm that can decomposes signal into a linear expansion of waveforms that belong to a redundant dictionary of functions, these waveforms are selected in order to best match the signal structures. For a particular signal, according to the characteristics of the signal, the algorithm can choose the best spread functions adaptively. By using less function more accurate represent the signal, it can decompose a signal into coherent components. The new feature extraction of the fault signal method in the neutral indirectly grounded system which is based on the atomic sparse decomposition.

\section{Time-Frequency Atom Dictionaries}

Time-frequency signal decomposition such as Fourier transforms and wavelet transforms has been used for many years. It is often essential to adapt the time-frequency decomposition to the particular signal structures to extract information from complex signals. The algorithm provided in this paper can decomposes signal into a linear expansion of waveforms that belong to a redundant dictionary of functions, the selected waveforms are the best match of the signal structures. For a particular signal, the algorithm can choose the best spread functions adaptively on the basis of the characteristics of the signal. The functions more accurate to represent the signal are called atom, the possibly redundant dictionary of functions called atoms.

The matching pursuit algorithm is often used in the atomic decomposition process, it is a greedy adaptive decomposition to decompose signal into a linear expansion of waveforms that belong to atoms. The selected atoms have good time-frequency characteristic and can represent the inherent characteristics and critical information of the signal.

\subsection{The Gabor Dictonary}

Decomposing a signal over a redundant dictionary improves the compression efficiency, especially at low bit rate where most of the signal energy is captured by only few elements. We often use Gabor dictionary in atomic sparse decomposition. Define

$$
g_{\gamma}(t)=\frac{1}{\sqrt{s}} g\left(\frac{t-\tau}{s}\right) e^{j \xi t}
$$

The real Gabor atoms:

$$
g_{\gamma}(t)=\frac{K_{\gamma}}{\sqrt{s}} g\left(\frac{t-\tau}{s}\right) \cos (\xi t+\phi)
$$

$g(t)=2^{\frac{1}{4}} e^{-\pi t^{2}}$ is Gaussian window function, $\gamma=(s, \tau, \xi, \phi)$, $\gamma$ is the index of $g_{\gamma}(t), s$ is Scaling parameters, $\tau$ is Displacement parameters, $\xi$ is Frequency parameters, $\phi$ is Phase parameters. Such atomic space is boundless, and in practice, we can't search a boundless space, so the atomic database should be dispersed. $\gamma=\left(a^{j}, p a^{j} \Delta \tau\right.$, $k a^{-j} \Delta \xi$ ), while $a=2, \Delta \tau=1 / 2, \Delta \xi=\pi, 0<j<\log _{2} N$, $0<p<N 2^{-j+1}, \quad 0<k<2^{j+1}$. Then we can get a Gabor dictionary

$$
\begin{aligned}
& g_{r d}(n)=g_{j}\left(n-p 2^{j}\right) \cos \left(n k \pi 2^{1-j}+\phi\right) \\
& g_{j}(n)= \begin{cases}\delta(j) & j=0 \\
K_{\gamma d} g\left(n 2^{-j}\right) & j \in[1, L) \\
\frac{1}{\sqrt{N}} & j=L\end{cases} \\
& n=0,1, \ldots, N-1
\end{aligned}
$$

\subsection{Matching Pursuits Algorithm}

In contrast to orthogonal transforms, over complete expansions of signals are not unique. The number of feasible decompositions is infinite, and finding the best solution under a given criteria is a NP-complete problem. In compression, one is interested in representing the signal to be coded with the smallest number of elements, which is in finding the solution with most of the energy on only a few coefficients. Matching Pursuit is one of the suboptimal approaches that greedily approximate the solution to this NP-complete problem [13].

Matching Pursuits algorithm, which is a greedy adaptive decomposition that has the potential of decomposing a signal into coherent components. 


$$
\left\{\begin{array}{l}
f_{x}^{0}=f \\
f_{x}^{m}=f_{x}^{m-1}-\left\langle f_{x}^{m-1}, g_{\gamma}^{(m)}\right\rangle g_{\gamma}^{(m)} \\
g_{\gamma}^{(m)}=\arg \max _{g_{\gamma}^{(i)} \in D}\left|\left\langle f_{x}^{m-1}, g_{\gamma}^{(i)}\right\rangle\right|
\end{array}\right.
$$

$f$ is the original signal. After m iterations, we decompose $\mathrm{f}$ into the concatenated sum

$$
f=\sum_{n=0}^{m-1}\left\langle f_{x}^{(n-1)}, g_{\gamma}{ }^{(n)}\right\rangle g_{\gamma}{ }^{(n)}+f_{x}^{(m)}
$$

If we stop the algorithm at this stage, the summation of (6) recovers an approximation of $\mathrm{f}$, with an error equal to $f_{x}^{(m)}$.

\subsection{Building a Dictionary for Power System Signals}

In a very simplistic way, power systems can be considered to be built from transmission lines, sources, and loads. Besides, we should add to this model the discontinuities due to circuit switching caused by operative maneuvers and by the protection system. The employed model is given by [14-16].

$$
\begin{aligned}
f(t)=\sum_{q=0}^{Q-1} A_{q} & \cos \left(2 \pi f_{q} t+\phi_{q}\right) e^{-\rho_{q}\left(t-t_{s q}\right)} \\
\times & {\left[\left(t-t_{s q}\right)-u\left(t-t_{e q}\right)\right] }
\end{aligned}
$$

where each component is a damped sinusoid represented by a six-tuple $\left(A_{q}, f_{q}, \rho_{q}, \phi_{q}, t_{s q}, t_{e q}\right)$, where $A_{q}$ is its amplitude, $f_{q}$ is the frequency, $\rho_{q}$ is the damping factor, $\phi_{q}$ is the phase, and ${ }^{e q}$ are the component starting and ending times. Note that the model in (7) by no means compactly represents "all” possible phenomena in power systems signals.

\section{Example Analysis}

\subsection{Harmonics}

The original analog signal model:

$$
\begin{aligned}
u(t)= & \cos (100 \pi t)+p(t) 0.4 \cos (700 \pi t+\pi / 6) \\
& +q(t) 0.2 \cos (1300 \pi t+\pi / 3) \\
p(t)= & \begin{cases}1 & 0.04 s \leq t \leq 0.25 s \\
0 & \text { otherwise }\end{cases} \\
\mathrm{q}(t)= & \begin{cases}1 & 0.1 s \leq t \leq 0.5 s \\
0 & \text { otherwise }\end{cases}
\end{aligned}
$$

The total time is $0.5 \mathrm{~s}$, Adding seven times harmonic at $0.04-0.25 \mathrm{~s}$; 13th harmonic at $0.1-0.5 \mathrm{~s}$, sampling at $4 \mathrm{KHz}$.

Figure 1(a) shows the Waveforms of decomposed atoms, and the Table $\mathbf{1}$ shows the atom parameters of each iteration. From the Figure 1(a) we can see that the atomic decomposition has iterated only three times to separate the fundamental component and harmonic component effectively, and the feature extraction of the Correlative Component is obtained. Figure 1(b) shows that the decomposed signal is closed to the original signal, and the error of this method reached 10-3, continuing decomposition can make the approximate degree higher. Besides, the data in Table 1 shows that the frequency, phase angle, and the starting and termination time got from the atomic decomposition are basically identical to the original signal.

\subsection{Earth Fault Line Selection}

1) Put up a model of $10 \mathrm{kV}$ distribution network with PSCAD. As Figure 2 shows, there are four lines named $\mathrm{m}, \mathrm{n}, \mathrm{w}, \mathrm{p}$. The single-phase- to-ground fault happened at $0.5 \mathrm{~s}$. Collecting and analyzing the zero sequence current in the four lines.
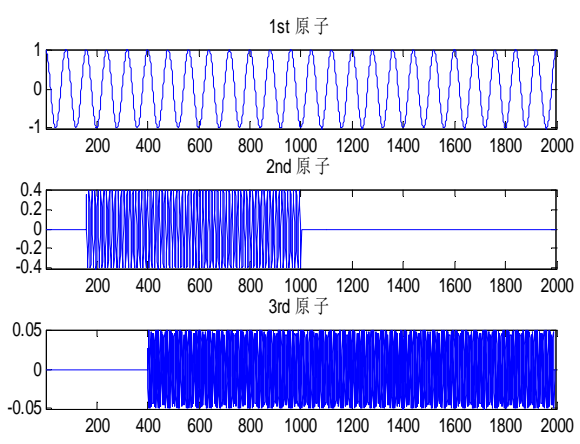

(a)
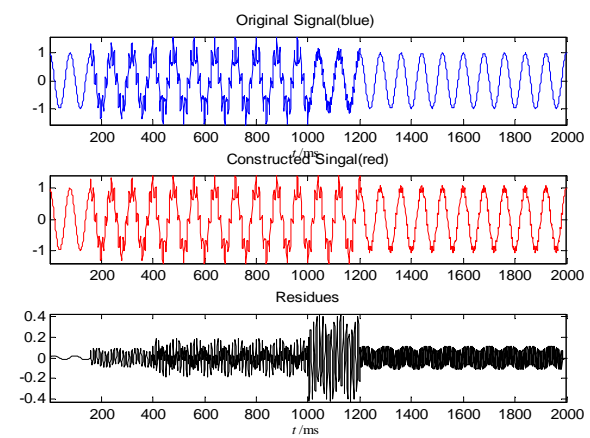

(b)

Figure 1. (a) Waveforms of decomposed atoms; (b) the reconstruction for harmonics.

Table 1. Decomposed atom parameters of signal.

\begin{tabular}{cccccc}
\hline Atom & $\begin{array}{c}\text { Amplitude } \\
(\mathrm{V})\end{array}$ & $\begin{array}{c}\text { Frequency } \\
(\mathrm{Hz})\end{array}$ & $\begin{array}{c}\text { Phase Angle } \\
(\mathrm{rad})\end{array}$ & $\begin{array}{c}\text { Start Time } \\
(\mathrm{s})\end{array}$ & $\begin{array}{c}\text { Terminal } \\
\text { Time(s) }\end{array}$ \\
\hline 1ed atom & 0.999 & 50.0000 & 0 & 0 & 0.5 \\
2rd atom & 0.388 & 350.0000 & 0.524 & 0.04 & 0.25 \\
3rd atom & 0.200 & 650.000 & 1.075 & 0.1 & 0.5 \\
\hline
\end{tabular}




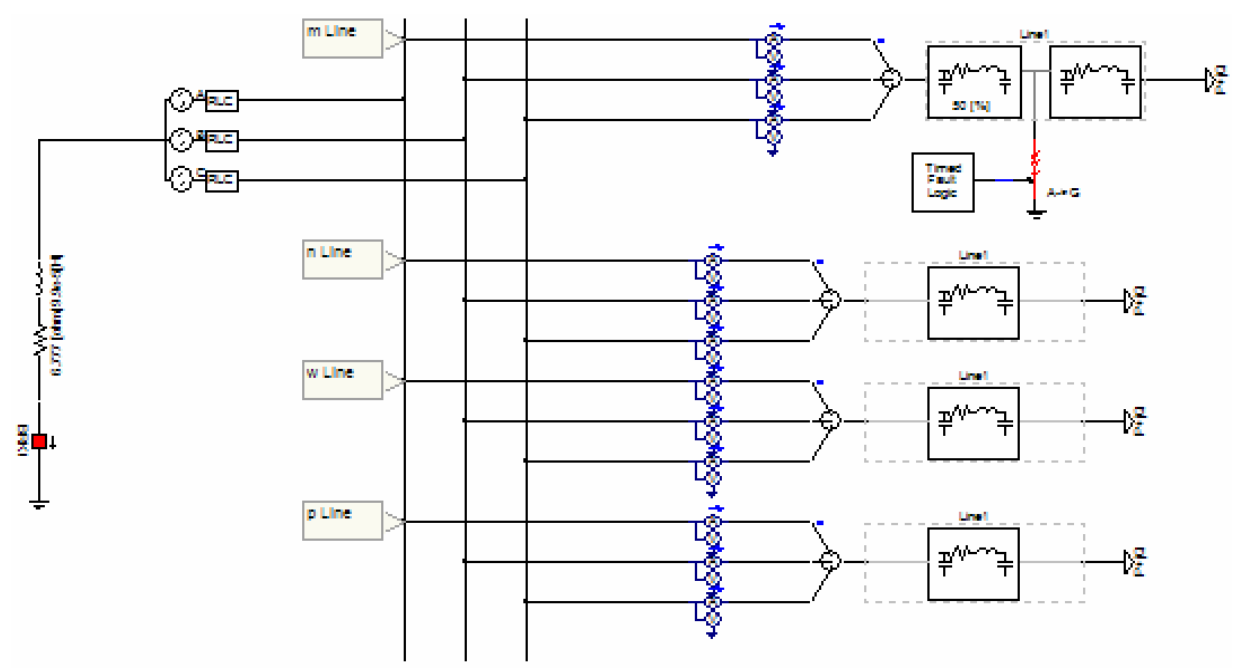

Figure 2. The model of $10 \mathrm{kV}$ distribution network.
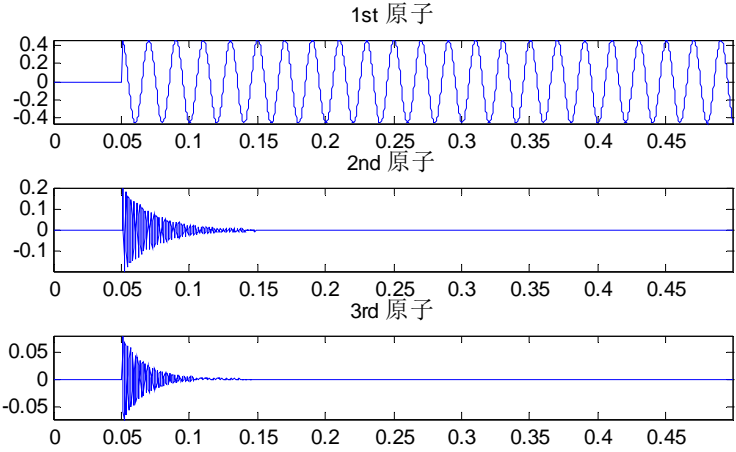

(a)
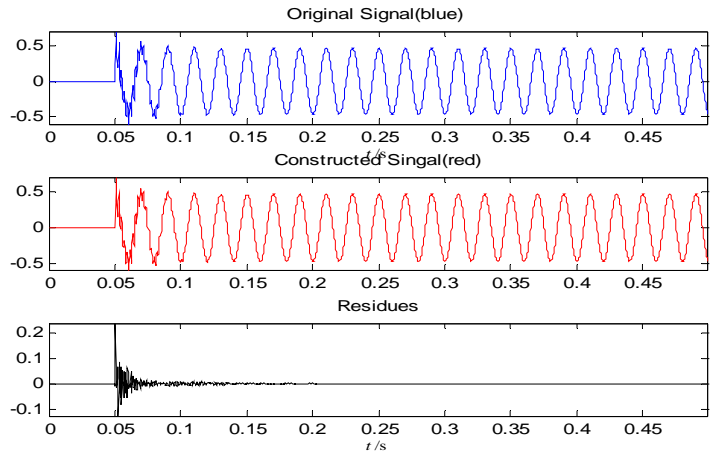

(b)

Figure 3. (a) Waveforms of decomposed atoms; (b) the reconstruction for zero sequence transient current of $\mathbf{m}$ line.

Table 2. Decomposed atom parameters of signal.

\begin{tabular}{ccccc}
\hline Line & Atom & $\begin{array}{c}\text { Amplitude } \\
\text { (V) }\end{array}$ & $\begin{array}{c}\text { Frequency } \\
\text { (Hz) }\end{array}$ & $\begin{array}{c}\text { Phase } \\
\text { Angle(rad) }\end{array}$ \\
\hline \multirow{2}{*}{ m line } & 2ed atom & 0.2048 & 488.08 & -5.6284 \\
& 3rd atom & 0.0812 & 547.03 & 26.8752 \\
\multirow{2}{*}{ n line } & 2ed atom & 0.1320 & 487.87 & 179.0498 \\
& 3rd atom & 0.0603 & 549.71 & -209.0478 \\
\hline
\end{tabular}

2) It is clearly observed from Figure 3(a) that the atomic decomposition can separate the fundamental component and harmonic component effectively with only three iterations. Figure 3(b) shows that the decomposed signal is similar to the original signal. From Table $\mathbf{2}$ we can see that the phase angles of the second atom is opposite to the third atom. Through which we can identify Single-Phase-to-Ground Fault line.

\section{Summary}

By the above analysis of the actual case, we apply a compression algorithm for signals measured during power system disturbances that obtains good effect while preserving important features for signal analysis. The damped sinusoidal dictionary by no means compactly represents "all” possible phenomena in power systems signals. Adaptive, analyticity and the sparse of Atomic decomposition make the algorithm has obvious advantages in power system fault line selection. Therefore, the method is feasible and practical, and can be applied broadly in power system.

\section{REFERENCES}

[1] S. Mallat and Z. Zhang, "Matching Pursuits with Time Frequency Dictionaries," IEEE Transaction on Signal Processing, Vol. 41, No. 12, 1993, pp. 3397-3415. doi:10.1109/78.258082

[2] X. J. Zeng, X. G. Yin, Z. Zhang, et al, "Study on Feeder Grounding Fault Protection Based on Zero Sequence Admittance," Proceedings of the CSEE, Vol. 21, No. 4, 2001, pp. 5-10.

[3] Z. Qi and Y. H. Yang, "Analysis of Technology of Fault 
Line Selection for Single-to-earth Faults in Neutral Point Non-effectively Grounded System," Automation of Electric Power Systems, Vol. 28, No. 14, 2004, pp. 1-5.

[4] H. C. Shu, J. Liu, D. J. Si, et al., "New Adaptive Method for Fault Line Selection in Non-solidly Grounded System," Automation of Electric Power Systems, Vol. 29, No. 13, 2005, pp. 64-68.

[5] Z. X. Zhang, S. H. Miao, X. N. Lin Xiangning and P. Liu, "Fault Line Selection Algorithm in Small Current Grounding Systems Based on à Trous Algorithm,” Automation of Electric Power Systems, Vol. 35, No. 1, 2011, pp. 67-70.

[6] X. Z. Dong and J. G. Bi, “Analysis on Transient Traveling Wave and Study on Fault Line Selection for Distribution Lines," Proceedings of the CSEE, Vol. 25, No. 4, 2005, pp. 1-6.

[7] F. Zhang, Z. C. Pan, H. F. Zhang, et al., "Fault Line Selection in Non-Solidly Earthed Network Based on Direction Travelingwave,” Proceedings of the CSEE, Vol. 27, No. 34, 2007, pp. 70-75.

[8] Q. Q. Jia, Q. X. Yang and Y. H. Yang, "Fusion Strategy for Single Phase to Ground Fault Detection Implemented Through Fault Measures and Evidence Theory," Proceedings of the CSEE, Vol. 23, No. 12, 2003, pp. 6-11.

[9] J. F. Dai, Y. X. Zhang, "Study on Adaptively Choosing Fault Line Under Single-Phase to Ground Fault Based on Analysis of Multi-Frequency Bands," Proceedings of the CSEE, Vol. 23, No. 5, 2003, pp. 44-47.

[10] Y. N. Wang, B. L. Huo, H. Wang, et al., A New Criterion for Earth Fault Line Selection Based on Wavelet Packets in Small Current Neutral Grounding System,” Proceed- ings of the CSEE, Vol. 24, No. 6, 2004, pp. 54-58.

[11] B. H. Zhang, H. M. Zhao, W. H. Zhang, et al., "Faulty Line Selection by Comparing the Amplitudes of Transient Zero Sequence Current in the Special Frequency Band for Power Distribution Networks," Power System Protection and Control, Vol. 36, No. 13, 2008, pp. 5-10.

[12] H. C. Shu and S. X. Peng, “A Fault Line Detection Algorithm for Distribution Network of Overhead Line and Underground Cable Mixed Lines Using S-transform Energy from Short Window Data," Transactions of China Electrotechnical Society, Vol. 24, No. 10, 2009, pp. 152-159.

[13] P. Frossard, P. Vandergheynst and R. M. Figueras, “A Posteriori Quantization of Progressive Matching Pursuit Streams," IEEE Trans on Signal Processing, Vol. 52, No. 2, 2004, pp. 525-535. doi:10.1109/TSP.2003.821105

[14] L. Lovisolo, E. A. B. da Silva, M. A. M. Rodrigues and P. S. R. Diniz, "Coherent Decompositions of Power Systems Signals Using Damped Sinusoids with Applications to Denoising,” in Proc. IEEE ISCAS, Scottsdale, AZ, Vol. V, 2002, pp. 685-688.

[15] M. M. Goodwin and M. Vetterli, "Matching Pursuit and Atomic Signal Models Based on Recursive Filter Banks," IEEE Transations on Signal Processing, Vol. 47, No. 7, 1999, pp. 1890-1902. doi:10.1109/78.771038

[16] L. Lovisolo, E. A. B. da Silva, M. A. M. Rodrigues and P. S. R. Diniz, "Efficient Coherent Adaptive Representations of Monitored Electric Signals in Power SystemsUsing Damped Sinusoids," IEEE Transations on Signal Processing, Vol. 53, No. 10, 2005, pp. 3831-3846. doi:10.1109/TSP.2005.855400 